\title{
Is CBR Applicable to the Coordination of Search and Rescue Operations? A Feasibility Study
}

\author{
Irène Abi-Zeid ${ }^{1}$, Qiang Yang ${ }^{2}$, and Luc Lamontagne ${ }^{1}$ \\ ${ }^{1}$ Defence Research Establishment Valcartier, 2459 boul. Pie-XI, \\ Val Belair, Quebec, G3J 1X5, Canada \\ \{irene.abi-zeid, luc.lamontagne\}@drev.dnd.ca \\ ${ }^{2}$ Simon Fraser University, School of Computing Science \\ Burnaby, British Columbia, V5A 1S6. Canada \\ qyang@cs.sfu.ca
}

\begin{abstract}
In response to the occurrence of an air incident, controllers at one of the three Canadian Rescue Coordination Centers (RCC) must make a series of critical decisions on the appropriate procedures to follow. These procedures (called incident prosecution) include hypotheses formulation and information gathering, development of a plan for the search and rescue (SAR) missions and in the end, the generation of reports. We present in this paper the results of a project aimed at evaluating the applicability of CBR to help support incident prosecution in the RCC. We have identified three possible applications of CBR: Online help, real time support for situation assessment, and report generation. We present a brief description of the situation assessment agent system that we are implementing as a result of this study.
\end{abstract}

\section{Introduction}

In response to the occurrence of an air incident, controllers at one of the three Canadian Rescue Coordination Centers (RCC) must make a series of critical decisions on the appropriate procedures to follow in order to deal with the incident. These decisions and procedures (called incident ${ }^{1}$ prosecution) include an assessment of the degree of emergency, a formulation of the hypotheses on what might have happened and where, the development of a plan for the search and rescue (SAR) missions and in the end, the generation of reports. The workflow of a controller may be roughly described as follows:

1. Receive alert;

2. Classify the situation through an interactive $\mathrm{Q} / \mathrm{A}$ process;

3. Iteratively narrow down the range of hypotheses by gaining new information through the information gathering process (communications search);

${ }^{1}$ The term usually used in a RCC is case prosecution. However, to avoid confusion with a case in $\mathrm{CBR}$ we will use the term incident prosecution. 
4. Initiate search planning;

5. Further narrow down the hypotheses using the new information gained;

6. Initiate SAR missions, task resources and monitor progress. At the same time, record important events;

7. Generate report.

It is important to note that not all air incidents lead to search planning. Incident prosecution is often limited to receiving the alert, classifying the situation, narrowing down the range of hypotheses (steps 1 to 3 ) and generating a report (7).

Prosecuting a SAR incident is knowledge intensive and exhibits strong real time characteristics. A typical controller handles two to three incidents at the same time. Furthermore, the Canadian RCCs receive well over 5,000 incidents per year. It would therefore be beneficial to develop a decision support system for automating as much of the process as possible, and for capturing and reusing the knowledge. Cottam et al. [2], [3] describe work done in the UK on a generic knowledge acquisition approach for search and rescue planning and scheduling operations. The authors found that the SAR problem solving is structured enough to allow a decision support system to advise the human controller.

Having such a system would enable RCC controllers to better support and coordinate their incident prosecution in real time, to streamline the incident reporting procedures, and to help train junior operators using realistic SAR scenarios. As part of an effort to design decision aid tools for the RCC controller, we decided to investigate and evaluate the applicability of CBR to help support incident prosecution in the RCC.

We begin in section 2 by describing the incident prosecution process. Section 3 presents our approach for evaluating the applicability of CBR to the RCC environment. This includes a brief description of the interviews conducted with the controllers as well as a summary of the related documents and databases surveyed. Section 4 presents our findings and recommendations on how CBR could be used, and section 5 presents the agent system that we are developing as a result of this study. We conclude in section 6 .

\section{Incident Prosecution in the RCC}

In general, SAR incident prosecution can be broken down into three phases where each phase can somewhat overlap the adjacent ones. For example, when a telephone call is received (first notice), the operation enters an uncertainty phase. In this phase the controller will collect information about the details of the flight plan and people involved. If not enough information has been obtained, the operation will progress into the alert phase whereby the controller will expand the information gathering activities and alert SAR agencies. If a certain amount of time has passed after the uncertainty phase without obtaining more information about the plane, the operation enters the distress phase. In this phase the controller initiates the tasks that consist of planning, coordinating, and monitoring search missions. Once the cause of the incident and the location of the aircraft are determined, he may be required to 
mobilize and monitor the rescue process. Tasks in this phase involve notifying various agencies involved, including hospitals and police, notifying the relatives and dispatching planes or helicopters to the crash site. An incident report will be filed in the end, and news dispatches will be sent out to various media agencies before the incident is closed.

The tasks conducted in the uncertainty and alert phases are called situation assessment (SA), a process similar to diagnosing a patient by a doctor. SA refers to the tasks of finding out the true nature of the incident by formulating and verifying hypotheses through an information gathering process. It is much like detective work. Over $90 \%$ of the incidents are false alarms, caused by faulty equipment, power lines or even Electronic Locator Transmitters (ELT) that the pilots forgot to turn off. Furthermore, the receipt of reports on an overdue (late) aircraft does not necessarily imply that the aircraft itself has crashed somewhere. Rather, there are many possibilities why the craft has gone missing, ranging from the fact that the pilot may have landed halfway to refuel, to the possibility that the pilot never took off in the first place. To summarize, the decision process in incident prosecution consists of situation assessment and mission planning and monitoring. Figure 1 presents a schematic description of the controller's tasks.

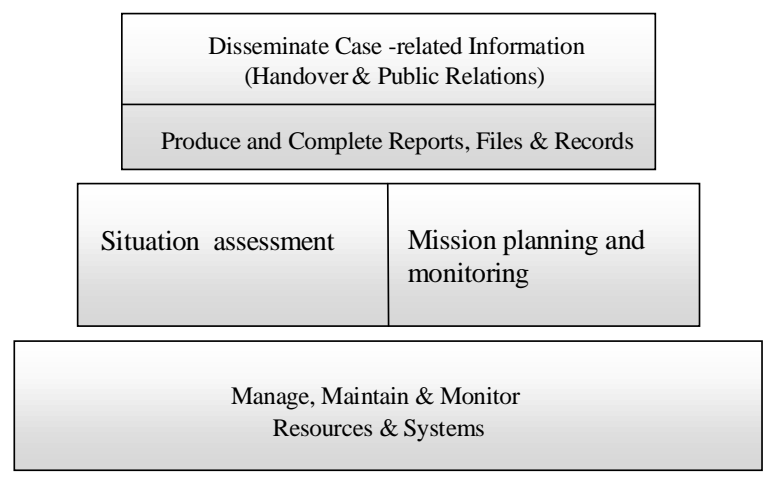

Fig. 1. Schematic description of a controller's tasks

\subsection{Why CBR?}

In the past, CBR has been applied to areas similar to our problem. These include classification, diagnosis and planning. The PROTOS system is used for classifying hearing disorders [12]. Based on the knowledge on a given patient, PROTOS finds a similar case and uses that case's class to determine the patient's disorder type. The CASEY system is designed to obtain a causal explanation regarding a patient's disorder given his signs and symptoms [8]. The CHEF system was designed to suggest recipes for cooking [5]. Given the goals of the meal, including style and ingredients, CHEF produces a sequence of actions by modifying a previously used recipe. It indexes the failures it has encountered in the past and uses domain specific knowledge to address these failures. MI-CBP is a Mixed-Initiative Case-Based planning system that is the integration of two systems: ForMAT, a case-based system 
for supporting military force deployment planning within the military transportation domain and Prodigy/Analogy for automating plan construction or adaptation [17].

From our initial knowledge of the SAR domain, we felt that there was at least one possibly worthwhile application for CBR: Situation assessment. When the first notice is received, a controller could retrieve from a case base cases with similar initial information. He then could adapt the past problem solving strategies regarding hypotheses formulation and information gathering. We have therefore concentrated in this study on situation assessment and excluded the mission planning and monitoring part of the controller's tasks.

\section{Our Approach}

The initial questions that we asked are:

- What is a case? Can a RCC incident be considered a CBR case?

- Do controllers make use of past incidents in their operations (implicitly or explicitly)?

- Can the incidents be compared, generalized?

- Is there sufficient historical information? And can the information be exploited by CBR techniques?

- Is it possible to quantify similarity and dissimilarity?

- Is the knowledge of a controller more rule-based or case-based?

In order to try to answer these questions, we went through a series of interviews as well as through the documents and the databases that consigned the historical RCC incident information. We summarize our observations below.

\subsection{Interviews}

Our first task was to get familiar with the RCC operations and the accumulated data. This was accomplished through visits to the RCCs and interviews with controllers: Six visits to RCC Victoria over a six months period, a review of four days of taped interviews with RCC Trenton, individual discussions with experienced controllers on other occasions, and participation in a one-week training course provided to controllers. This allowed us to gain first hand experience in actual operations of the $\mathrm{RCC}$, and to assess the dataflow and workflow of the organization.

During the first visit, an overview of the operations of the RCC was given by a senior controller. This was followed by an overview of CBR given by us. We had prepared a set of questions for the interviews. These were mostly related to the activities of a controller. We then asked about the training required and some important factors that make a successful controller. We learnt that incidents are currently being recorded on paper in real time, using a checklist and tables. In the future, a database front-end system (CaseMaster) will be used to record all incident 
information, including the major actions taken by the RCC controllers and the time and circumstances of the action. This system has been tested and will be used in the near future.

During our various meetings, the controllers expressed the following concerns: First, it is very time consuming to brief or communicate with another controller in the $\mathrm{RCC}$ or at another RCC about the current incident information. Second, it is time consuming to file reports at the end. Third, it is sometimes possible to forget factors that should be evaluated while narrowing down potential hypotheses especially in the presence of incomplete information. They felt that recorded incidents could help address these problems. They rejected a rule-based system as a potential decision aid because they felt it was too "rigid". Furthermore, they confirmed that they retrieved similar incidents and solved new problems by making use of past incidents, especially for device related analogies. For example, if an incident involves a Beaver plane, then past knowledge about the most breakable devices on that plane can serve as a hint for the possibilities for the current incident.

\subsection{Procedures Manual}

In order to complement our knowledge acquisition process, we studied the national SAR manual [11] and RCC Victoria Standard Operations Procedures [14] for carrying out SAR operations in Canada. One of the most interesting aspects of the manuals is a collection of possible scenarios and their corresponding solutions. It is interesting to note that this method of presenting scenario-solution pairs is consistent with the problem-solving model of CBR.

\subsection{Historical Data}

We examined the RCC Victoria Statistical Summary [15]. This 8-page document reports on the annual operational figures. It begins with a national comparison of the incidents that occurred in 1996. It then separately reports on the use of assets and resources for marine, air and land SAR incidents in 1996. The summary is very useful in providing a big picture of the SAR incidents handled at RCC Victoria and the corresponding statistical figures, however it is not of any practical use for CBR.

\subsection{Incident Logs}

As incidents are prosecuted, information is recorded in incident logs containing incident descriptions, unit assisted descriptions, type of incident, difficulty level, action taken, weather report, resources used, critical factors and anomalies, etc. This information will be recorded in electronic form in the near future. The most interesting aspect of these forms is the manual logging of pertinent chronological descriptions of the actions taken by the controllers. 


\subsection{Statistical Database Model SISAR}

When the operations are completed, information is logged into SISAR, a statistical database which keeps information similar to the one contained in the incident logs. However, SISAR only provides categorical summaries of an incident, recording information such as the number of persons on board and the type of aircraft involved. It does not record all the relevant causal factors and the process followed by a controller to narrow down the hypotheses. Our study concluded that not enough detail is provided in the SISAR database logs about the events that occur and the reasoning that goes on.

\section{Results}

In studying the SAR domain and investigating the application of CBR, we focused on the following issues [7], [9]:

1. What is a case?

2. What are the indexes?

3. How are the cases acquired?

4. Is there any adaptation?

5. What are some recommendations?

Our results are summarized in the form of answers to each of the above questions. In defining what is a case, we kept in mind that a case normally consists of the following elements:

- Name and id;

- Keywords for retrieval;

- Facts denoting problem solving context;

- Solution used in the past;

- Outcomes denoting success or failure;

- Solution context;

- Interpretations and annotations of the case;

- Links to other cases

\subsection{CBR for Online Help}

The first envisaged application of CBR was to provide online help with procedures during real time incident prosecution. This is similar to using CBR for help desks in 
technical troubleshooting. The main purpose of such a system would be to remind the controller of the appropriate procedures when the controller is aware of the stage in which the SAR operation is. For example, when a controller is aware that he is in the uncertainty phase and would like to consult the operational procedures as outlined in the National SAR Manual.

Description of a Case. In this application, a case would consist of a pair, where the first element of the pair is a problem resolution context or phase (for example, aircase-uncertainty-phase), and the second element is a procedure itself that may be presented in textual format on the computer screen. As an example, consider the procedure for a distress phase operation taken from [11].

Case name: Distress phase of Air SAR

Case content: RCC action during DISTRESS phase of an aircraft emergency:

- Initiate action with appropriate SAR units and services;

- ...

- When the incident involves an aircraft of foreign registry, RCC shall inform National Defense Operations Center to advise appropriate embassy if required;

- Develop rescue plan if casualties require assistance, notify medical facilities, police/coroner, establish the most expeditious means and method of rescue.

Description of the Indexes. The indexes for these cases are all the relevant information that one can use to classify the current situation in terms of phase information. In the air SAR phase identification example, the indexes can be the following questions:

- Aircraft didn't land on time and lost communication? (yes/no);

- ...

- Following the uncertainty phase, communications search received no new information? (yes/no);

Acquiring the Cases and Using Adaptation. Major sources of the case information are the SAR manuals and the training manuals. There are probably simple forms of adaptation that can be performed on these procedures, although in the current practice, these adaptations are mostly done by the controllers.

Recommendations. Our observations are that the experienced controllers have already mastered all the basic procedures indicated in the SAR manuals. This assertion is based on the fact that they are actually doing their job in the operational environment. We suspect that this method of using CBR where cases are recorded as operational procedures would be limited to training and to providing assistance to junior air SAR controllers. 


\subsection{CBR for Situation Assessment}

We present here the most promising application of CBR to incident prosecution: Situation assessment. Recall that the first part of incident prosecution involves finding out which of the hypotheses holds for the current situation. A hypothesis is a plausible cause and outcome for the incident. An example of a hypothesis on a cause of incident is "mechanical failure" for the outcome "crash". A lot of information must be gathered by the RCC controller in order to narrow down the hypotheses space. For example, the controller may check the weather condition to see whether the likelihood of a crash is large given an overdue report. At the same time, a request will be sent out to get the flight plan of the pilot in order to find all airports where the pilot might have landed, and so on. CBR can be used to rank and eliminate various hypotheses and to determine the associated information gathering tasks.

Description of a Case. A case may consist of each possible hypothesis (cause, outcome). It consists of both the problem attributes and the associated methods to operate on them. In particular, a case here would consist of the following elements:

- A hypothesis for the possible cause and possible outcome of the incident;

- A hierarchical task network (HTN) [4], [16] for the information gathering process to confirm the hypothesis (Fig. 2);

- A record of executed information gathering tasks for the current hypothesis object, and the information gathering steps yet to be executed for further confirming the hypothesis;

- An evaluation function of priorities for not-yet executed information gathering tasks;

- Indices with weights attached to the expected values of the answers to the queries.

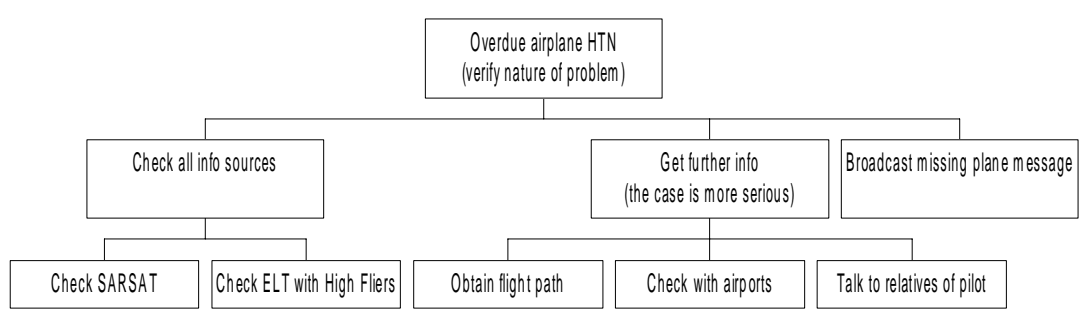

Fig. 2. An information gathering HTN example for an overdue airplane

Description of the Indexes. For the controller to assess the current situation, rank the remaining hypotheses, and weigh the next steps, a channel must exist between the known facts and knowledge and the system stored HTNs and hypotheses. This 
channel is provided by a layer of indexes. The cases may be indexed by different problem features:

- Overdue planes;

- ELT signals;

- Flare sightings;

- Crash reports;

- Mayday calls;

- Problem context;

- Weather reports;

- Vehicle involved;

- People involved;

- Fight path and location information.

Acquiring the Cases and Using Adaptation. Cases can be acquired through three sources. First, the National SAR Manual and other written documents provide a detailed outline of procedures and possibilities for the air SAR causes. Second, additional causes and hypotheses can be obtained from the RCC controllers and pilots themselves. The controllers we have met all have vast amount of experience on a potential range of hypotheses. Third and most importantly, causes can be obtained from a systematic scanning of the incident logs. The adaptation is in the form of selecting a task in an HTN to expand, and in adding and deleting new tasks in the HTN of a retrieved case. To expand a task, one has to determine which subtasks to execute and which information sources to access given several alternatives. Choosing an appropriate alternative will have an important impact on the effectiveness of the SAR operation.

Recommendations. There are two ways to use CBR in the context of a case as defined throughout this section. The first envisaged way was to have an interactive system in the form of a checklist into which the controller records his information gathering actions and the results. These results are then used by the system to update the list of the information gathering tasks that remain to be executed either by the controller or automatically when possible. This approach has the limitation of involving the controllers extensively in real time for providing values for indexes. It would therefore be difficult to win over the support of the controllers for such a system.

The second envisaged way, (and the one currently under development) is to use case-based HTN retrieval as a reminder list in the background and as means to acquire automatically the information that is available electronically. Information gathering is seen here as a planning task [6], [13]. The resulting system would be in the form of a background intelligent agent with minimum interaction with the controller. A limitation with this approach is that a fairly sophisticated monitoring and 
filtering system that reports on relevant incoming information must be assumed for telephone sources.

\subsection{CBR for Report Generation}

We present here the third possibility for using CBR: Raw CBR cases for report generation.

Description of a Case. A case in this approach would be a full recording of the history of events unfolding along a time line. An example is shown below:

Begin case:

Case 009:

Indexes:

Weather condition: clear

Caller: Airport staff

Table 1. An example of a chronological list of actions and events.

\begin{tabular}{|l|l|}
\hline Events Time & Events \\
\hline 10:23am & $\begin{array}{l}\text { Call received about an overdue plane: } \\
\text { Information: small plane; expected landing } \\
\text { time 9 am; Person On Board unknown; }\end{array}$ \\
\hline 10:25am & Called to obtain flight path plan information \\
\hline $10: 30$ & Called to talk to wife of pilot \\
\hline 10:30am & $\begin{array}{l}\text { Electronic Locator Transmitter (ELT) signal } \\
\text { received along flight path }\end{array}$ \\
\hline
\end{tabular}

Forgotten Tasks:

A call to RCMP should have been initiated at 10:25am.

A request for more information should have been sent out to airports at source and destination.

Pitfalls to be avoided

Wait for more information before phoning the wife.

Outcome

Plane landed to refuel in an airport along the flight path. End Case.

Description of the Indexes. The indexes for this case base would be a combination of the initial triggers for the case, and the contextual information such as the weather report and the type of airplane. As such, the set of indexes is not very different from those outlined in subsection 4.2.

Acquiring the Cases and Using Adaptation. One of the most promising methods would be to use an enhanced version of the CaseMaster system. In this situation, we are not looking at adapting the solutions to the incident but rather to adapt old reports 
based on the contents of the new incident. Here, CBR is used more as means for organizing, storing and retrieving incident logs.

Recommendations. In addition to generating reports, cases may be used as means of communication between controllers. Furthermore, they could serve as a basis for generating useful indexes for the application described in subsection 4.2. In this manner, we ensure that the case base index is always current and up to date.

\section{A Brief Description of ASISA: Agent System for Intelligent Situation Assessment}

We present in this section a brief description of an initial prototype to assist the controller in situation assessment. This is the result of the second recommendation. This tool, ASISA: Agent System for Intelligent Situation Assessment, is a combination of CBR and Hierarchical Task Network (HTN) planning techniques. In this initial prototype, cases are used to describe hypotheses and to encapsulate information-gathering queries for identifying the correct hypothesis. Figure 3 presents a schematic description of ASISA.

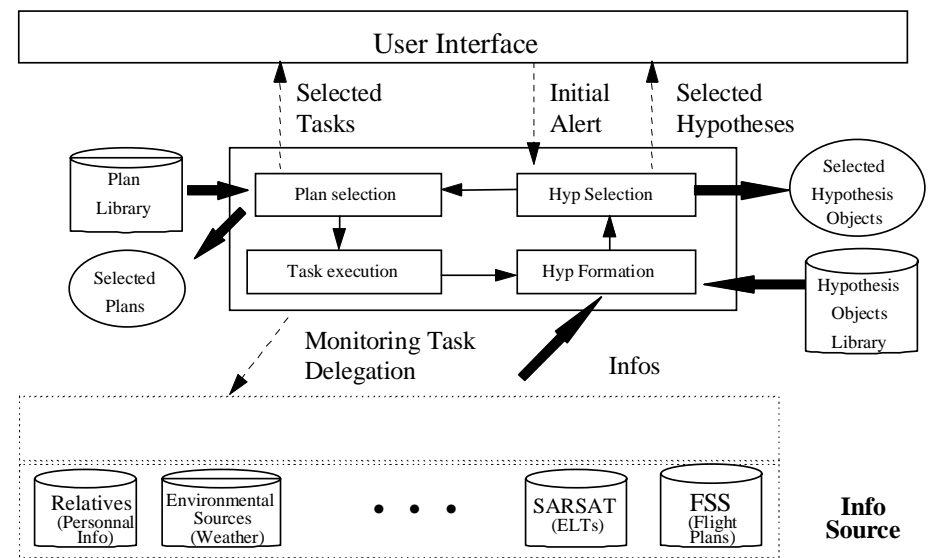

Fig. 3. A diagram illustrating the main components of ASISA

First, upon receiving an initial indication of a problem, the available relevant information is input into the system. The system retrieves a collection of similar cases from the case base. They consist of the hypotheses $\mathrm{H}_{1}, \mathrm{H}_{2}, \ldots \mathrm{H}_{\mathrm{n}}$ that can be used to characterize the current situation, where each of the $\mathrm{H}_{\mathrm{i}}$ 's provides a plausible cause for the current case such as "plane crashed due to mechanical failure". Subsequently, the system enters a cycle in which it identifies (from the case base) the information gathering tasks described by the HTN associated with each hypothesis object. These HTNs are refined by the plan selection module and used by the task execution module to determine the information gathering actions to be executed next. The process 
continues until a final conclusion about the nature of the incident is reached by the ASISA system. The overall workflow for this iterative process is depicted in Figure 4.

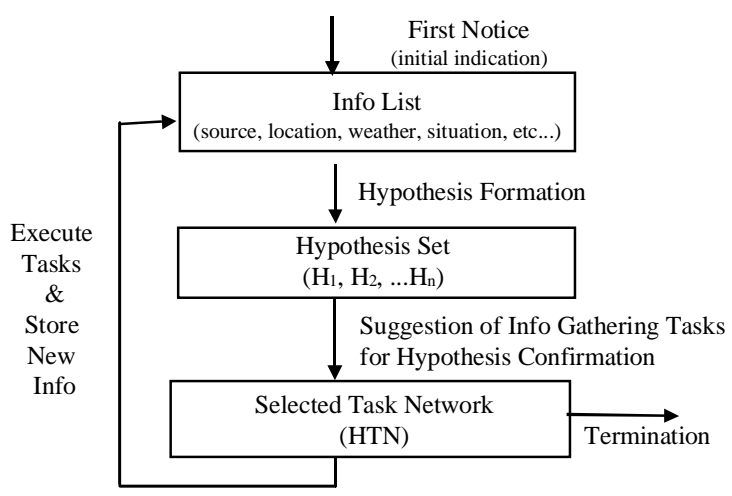

Fig. 4. Information flow in ASISA

The agent-based ASISA system will benefit the SAR controller in several ways. First, because the agent is constantly monitoring a variety of information sources, it can help filter out a large quantity of irrelevant information, and help the controller concentrate on the critical information only. Second, given the overwhelming workload during the high seasons for air incidents, the agent system can help improve the accuracy and shorten the time required for assessing a case. This effect translates directly into one of saving more lives. Third, for junior air SAR controllers, the agentbased system can become a handy decision support system and a tutoring system. We expect that the learning speed of the new controllers will be improved with the help of our agent system.

\subsection{Related Work}

HICAP (Hierarchical Interactive Case-based Architecture for Planning) [9] is a planning architecture developed to support planning of Noncombatant Evacuation Operations by assisting military commanders in interactively refining plans. It is similar to what is proposed in ASISA in the sense that it uses HTN to represent tasks and subtasks. However, a major difference with ASISA is that while HICAP is meant to be highly interactive, ASISA is meant to be very little intrusive and with minimum interaction with the controller. The reason is that incident prosecution is a real-time operation where the controller has no time to interact with a computer. Since most of the controller's activities are conducted over the phone, one of our ongoing research projects is to monitor telephone conversations and try to extract the information necessary to feed the ASISA system. However at this point, we assume that such information is readily available for ASISA.

Another application domain for ASISA has been identified as the cable TV troubleshooting domain that bears resemblance with the search and rescue problem domain [1]. 


\section{Conclusions}

Our study has revealed three possible ways to use a CBR system for incident prosecution, each corresponding to a different usage of the case information:

1. CBR for online help: A case may be viewed as a specific procedure on how to deal with a certain situation as outlined in the standard operational procedures;

2. CBR for situation assessment: A case may be viewed as a hypothesis on the cause and outcome of an incident, along with the information gathering tasks for ascertaining that cause;

3. CBR for report generation: A case may be viewed as a step-by-step recording of all actions taken by the controller.

As a result of this study, we are currently implementing ASISA, an agent system for situation assessment where CBR is used for storing, and retrieving hypothesis along with their associated information gathering tasks represented as HTNs.

Many issues still need to be explored with regards to CBR and SAR, mainly the applicability of CBR to support the second phase of incident prosecution: Mission planning and monitoring.

\section{References}

1. Carrick C., Qiang Y., Abi-Zeid, I., Lamontagne L.: Activating CBR Systems through Autonomous Information Gathering. ICCBR'99 (1999)

2. Cottam, H., Shadbolt, N., Kingston, J., Beck, H., Tate, A.: Knowledge Level Planning in the Search and Rescue Domain. In Bramer, M.A., Nealon, R. (eds.): Research and Development in Expert Systems XII. SGES Publications (1995) 309-326

3. Cottam, H., Shadbolt, N.: Knowledge Acquisition for Search and Rescue. Proceedings of the 10th Knowledge Acquisition for Knowledge Based Systems Workshop, KAW 96 (1996)

4. Erol, K. Hierarchical Task Network Planning: Formalization, Analysis and Implementation. Ph. D. Thesis, University of Maryland (1994)

5. Hammond, K. Case Based Planning: A Framework for Planning from Experience. Journal of Cognitive Science, 14 (3) (1990)

6. Knoblock, C. and Ambite, J.-L.: Agents for Information Gathering. In Bradshaw, J. M. (ed.): Software Agents. (1997) 347-374

7. Kolodner, J.: Case Based Reasoning. Morgan Kaufmann Publishers, Inc. (1993)

8. Koton, P.: Reasoning about Evidence in Causal Explanation. In Proceedings of the 1998 AAAI Conference. AAAI Press/MIT Press (1988)

9. Leake, D.: Case Based Reasoning: Experiences, Lessons and Future Directions. AAAI Press (1996)

10. Munoz-Avila, H., Aha D. W., Breslow L., Nau D.: HICAP: An Interactive CaseBased Planning Architecture and its Application to Noncombatant Evacuation Operations. NCARAI Technical Note AIC-99-002 (1999)

11. National SAR Manual. December (1995) 
12. Porter, B., Bareiss, R., and Holte, R.: Concept Learning and Heuristic Classification in Weak Theory Domains. Artificial Intelligence (45) (1990) 229263

13. Pryor, L. and Collins G.: Planning to perceive: A utilitarian approach. In Working notes of the AAAI Spring Symposium on the Control of Selective Perception. March (1992)

14. RCC Victoria Standard Operating Procedures (1997)

15. RCC Victoria 1996 Statistical Summary (1996)

16. Sacerdoti, E. D.: Planning in a hierarchy of abstraction spaces. In Allen, J., Hendler J., and Tate A. (eds.): Readings in Planning. Morgan Kaufman (1990) 98-108

17. Veloso, M. M., Mulvehill A. M., Cox M. T.: Rationale-Supported MixedInitiative Case-Based Planning. In Proceedings of the Ninth Conference on Innovative Applications of Artificial Intelligence. AAAI Press (1997) 1072-1085 